\title{
The role of transdermal estrogen sprays and estradiol topical emulsion in the management of menopause-associated vasomotor symptoms
}

This article was published in the following Dove Press journal:

International Journal of General Medicine

8 May 2010

Number of times this article has been viewed

\author{
Amy M Egras \\ Elena M Umland \\ Jefferson School of Pharmacy, \\ Thomas Jefferson University, \\ Philadelphia, PA, USA
}

Correspondence: Elena M Umland Associate Dean for Academic Affairs, Jefferson School of Pharmacy, Thomas Jefferson University, I $30 \mathrm{~S}$ 9th St, Suite 1520, Philadelphia, PA 19107, USA

$\mathrm{Tel}+\mathrm{I}(2 \mathrm{I}$ ) 503-9087

Fax +I (2I5) 503-9052

Email elena.umland@jefferson.edu

\begin{abstract}
Vasomotor symptoms (VMS) are among the most bothersome complaints of postmenopausal women. To date, the most widely studied and effective treatment for VMS is hormone replacement therapy, consisting of estrogen (in women without a uterus) or estrogen plus progestin (in women with a uterus). Traditionally, oral estrogens have been used for treatment. However, over the years, additional estrogen formulations have been developed including transdermal patches; vaginal rings, creams, and tablets; and injectable preparations. Two newer formulations are transdermal estrogen spray and estradiol topical emulsion. This review evaluates the current literature assessing the use of these two newer formulations for the treatment of VMS associated with menopause.
\end{abstract}

Keywords: menopause, vasomotor symptoms, transdermal estrogen spray, estradiol topical emulsion

\section{Introduction}

Although all women will be subject to menopause and the cessation of menses, not all women will experience its associated symptoms. Of the associated symptoms, vasomotor symptoms (VMS) are the most bothersome. They may include hot flashes (rapid onset of intense heat sensation, sweating, and flushing lasting approximately 5 to 10 minutes) and/or night sweats. ${ }^{1-3}$ They are the leading reason menopausal women seek medical care as can be illustrated by a 2002 Gallup poll of menopausal women showing that hot flashes, night sweats, mood disturbances, and sleep disturbances were the top four reasons for which medical attention was sought at this time in their lives. ${ }^{4}$ Overall, VMS are estimated to affect $14 \%$ to $51 \%$ of premenopausal women, $35 \%$ to $50 \%$ of perimenopausal women, and $30 \%$ to $80 \%$ of postmenopausal women. ${ }^{3}$

On average, most women experience VMS for 6 months to 2 years; however, approximately $10 \%$ of women report experiencing VMS for 10 or more years. ${ }^{4,5}$

In total, it is estimated that $60 \%$ of peri- and postmenopausal women seek care for their menopausal symptoms. ${ }^{6}$

\section{VMS}

There is currently no consensus on the pathophysiology of menopause-associated VMS; however several mechanisms have been proposed. One is a narrowing of the thermoregulatory threshold between sweating and shivering in the hypothalamus. ${ }^{7}$ This narrowing is thought to be caused by decreased levels of circulating serotonin, increased levels of norepinephrine, and/or decreased estrogen concentrations. ${ }^{1,5}$ The perimenopausal decline in ovarian estradiol production results in diminished negative-feedback effects 
on the anterior pituitary, leading to a compensatory increase in the secretion of leuteinizing hormone from the pituitary, a process regulated by gonadotropin-releasing hormone in the hypothalamus. ${ }^{8}$ Pulsatile surges of gonadotropin-releasing hormone due to estrogen deficiency affect the hypothalamic neurons that control central thermoregulation centers. ${ }^{9}$

It has also been hypothesized that the ratios of the specific types of estrogen (ie, estradiol or estrone) may be better correlated with the occurrence of VMS than the overall circulating level of estrogen. ${ }^{10}$ Compared to premenopausal women in whom the most abundant circulating estrogen is estradiol, the more potent form of estrogen, throughout the peri- and postmenopausal years, the most abundant circulating estrogen shifts to estrone, a much less potent form of estrogen. ${ }^{8}$ Further, the occurrence of VMS has been found to correlate better with an acute decline in estrogen levels than with the actual measured levels of estrogen. ${ }^{5}$

\section{Estrogen therapy (ET)}

Given the above proposed mechanisms for VMS, it is no surprise that hormone replacement therapy (HT), consisting of estrogen (in women without a uterus) or estrogen plus progestin (in women with a uterus [to protect against endometrial hyperplasia or cancer]), is the most widely studied and most effective treatment option for relieving VMS and is considered the standard of care for women with moderate-to-severe VMS. ${ }^{1,2}$ The AACE and ACOG recommend the use of HT at the lowest effective dose and for the shortest duration possible (preferably $\leq 5$ years) in women for whom the potential benefits outweigh the potential risks. ${ }^{1,11}$ The 5-year cut-off for HT is suggested because most women will experience spontaneous cessation of menopausal symptoms within 5 years of onset. ${ }^{1}$ Use of HT for longer durations may be appropriate in some women, such as those who judge the benefits of VMS relief to outweigh the potential risks after failing an attempt to discontinue HT, those with continued VMS who are also at high risk for osteoporotic fractures, and those requiring osteoporosis prevention who cannot take alternative therapies. ${ }^{2}$ Several weeks may be required to determine the efficacy of HT in treating VMS. ${ }^{5}$ Contraindications to the use of HT include breast or estrogen-sensitive cancers, undiagnosed vaginal bleeding, endometrial hyperplasia, blood clotting disorder, hypertension, liver disease, hypersensitivity to the active or excipient compounds in HT. ${ }^{1}$

The recommendation that HT is the standard of care for treating moderate-to-severe VMS is based on the large body of evidence demonstrating the effectiveness of estrogen or estrogen plus progestin in the reduction of VMS frequency and severity. In fact, a meta-analysis of 24 double-blind, randomized, placebo-controlled trials including a total of 3,329 subjects and ranging in duration from 3 months to 3 years demonstrated a significant $75.3 \%$ reduction in the frequency of hot flashes experienced per week (weighted mean difference, -17.92 , 95\% confidence interval $[\mathrm{CI}],-22.86$ to -12.99$)$ and a significant $87 \%$ reduction in the severity of symptoms (odds ratio [OR], 0.13; 95\% $\mathrm{CI}, 0.07$ to 0.23 ) relative to placebo. The most frequently used HT regimens noted in the meta-analysis included oral estradiol, conjugated equine estrogens, and ethinyl estradiol, with or without a 19 -nor testosterone derivative. ${ }^{12}$ These relative reductions are significant considering that the placebo response rate in this meta-analysis was $57.7 \%$. There are currently no data available to suggest that any one formulation of estrogen or estrogen plus progestin is clinically superior to another. ${ }^{5,11,13}$ Estrogen and progestin are available in various oral, transdermal (patch, spray, and emulsion), vaginal, and injectable preparations. Table 1 illustrates the recommended doses for these various products.

Table I Recommended dosing ranges for commonly used hormonal therapies used to treat vasomotor symptoms $\mathrm{s}^{1,2,5,14}$

\begin{tabular}{ll}
\hline Drug & Daily dose \\
\hline Estrogens & \\
Conjugated equine estrogens & $0.3-0.625 \mathrm{mg}$ \\
Micronized I7ß-estradiol & $0.25-1 \mathrm{mg}$ \\
Transdermal estradiol (patch) & $14-100 \mu \mathrm{g}$ \\
Transdermal estradiol (spray) & $1.53-4.59 \mathrm{mg}$ \\
Transdermal estradiol (emulsion) & $3.84 \mathrm{~g}$ \\
Ethinyl estradiol & $0.01-0.02 \mathrm{mg}$ \\
Vaginal estradiol ring & $0.05-0.1 \mathrm{mg}$ \\
Progestins & \\
Medroxyprogesterone acetate & $2.5 \mathrm{mg}$ (or $5 \mathrm{mg}$ for I0-14 days/month) \\
Micronized progesterone & $100 \mathrm{mg}$ (or $200 \mathrm{mg}$ for I0-14 days/month) \\
Norethindrone & $0.14-0.25 \mathrm{mg}$ (transdermal patch); 0.2-I mg (orally) \\
Levonorgestrel & $0.075 \mathrm{mg}$ \\
\hline
\end{tabular}




\section{ET dosage formulations}

Traditionally, ET has been administered orally. However, other routes of ET administration have been developed. Transdermal formulations, such as the patch, spray and topical emulsion, offer some advantages over oral estradiol administration. These formulations avoid hepatic first pass metabolism that is observed with the oral formulations and that can result in the variable bioavailability of estrogen and potentially the need for higher doses. In addition, these dosage formulations have little or no risk for the development of conditions such as hypertriglyceridemia, gallbladder disease, and thromboembolism..$^{15,16}$

However, while the transdermal administration bypasses first pass metabolism and results in effective therapy at lower estrogen doses, some concerns with this delivery system do exist. These include variable systemic absorption, skin irritation, and poor adhesion of the patches. ${ }^{17-19}$ Two newer formulations, transdermal spray and topical emulsion, may offer some advantages over both the orally administered and transdermal patch estrogen. This review examines the clinical data related to the use of the transdermal spray and topical emulsion ET to treat VMS associated with menopause.

\section{ET - transdermal spray and topical emulsion}

Presented here are summaries of the clinical trials evaluating the effects of transdermal estrogen spray and estradiol topical emulsion on VMS associated with menopause.

\section{ET - transdermal spray}

The transdermal estrogen spray is a clear spray that dries in 60 seconds and results in little to no skin irritation. ${ }^{20}$ It has been shown that significant transfer of estradiol via skin-to-skin contact does not occur; washing the application site 1 hour after application does not affect absorption; and applying sunscreen 1 hour prior to application also does not affect absorption. ${ }^{21}$

In a phase III, randomized, double-blind, placebo-controlled, parallel-group trial, Buster et al investigated the safety and efficacy of a transdermal estradiol spray. ${ }^{20}$ Postmenopausal women $(n=454)$ with at least 8 moderate-to-severe hot flashes per day (recorded in a daily diary) were randomly assigned to receive 1,2 , or 3 sprays of estradiol; or 1, 2, or 3 sprays of placebo to the inner forearm. One spray of estradiol delivered $1.52 \mathrm{mg}$ of estradiol. The primary efficacy endpoint was the change in frequency and severity ( 0 , none; 1 , mild; 2 , moderate; or 3 , severe) of moderate-to-severe hot flashes from baseline to weeks 4 and 12. For the 3 spray group, there was a reduction in the frequency of hot flashes per day of $-6.64 \pm 4.23$ at week 4 and a reduction of $-8.44 \pm 4.50$ at week 12 . This was statistically significant when compared to placebo $(P<0.001)$. Similar results were observed in the 2 spray group, with a frequency reduction of $-7.30 \pm 6.93$ at week $4(P=0.003)$ and $-8.66 \pm 6.65$ at week 12 $(P=0.010)$. There was also a statistically significant reduction of hot flashes from baseline in the 1 spray group with a frequency reduction of $-6.26 \pm 4.01(P=0.001)$ at week 4 and $-8.10 \pm 4.02$ $(P<0.001)$ at week 12 compared to placebo.

For the 3 spray group, changes in the severity score of the moderate-to-severe hot flashes decreased from $2.58 \pm 0.25$ at baseline to $2.15 \pm 0.73$ and $1.50 \pm 1.06$ at weeks 4 and 12 , respectively. These findings were statistically significant (week 4, $P=0.003$; week 12, $P<0.001$ ) when compared to placebo (baseline, $2.54 \pm 0.24$; week 4, $2.41 \pm 0.58$; week 12, $2.23 \pm 0.79)$. Similar results were observed in the 2-spray group with the severity score being $1.97 \pm 0.87$ at week 4 and $1.63 \pm 1.07$ at week 12 compared to a baseline score of $2.54 \pm 0.21$. These results were statistically significant (week $4, P=0.016$; week $12, P=0.041$ ) when compared to placebo (baseline, $2.54 \pm 0.21$; week 4, $2.29 \pm 0.71$; week 12, $2.00 \pm 0.96$ ). For the 1 -spray group, at week 12 , the severity decreased by -1.04 compared to $-0.26 \pm 0.60$ in the placebo group $(P<0.001)$ and there was a trend towards significance at week $4(P=0.057)$. It was also shown that $74.3 \%$ to $85.5 \%$ of women treated with transdermal estradiol spray had a $\geq 50 \%$ reduction in hot flash frequency when compared to baseline. The Greene Climacteric Scale scores, focusing on the vasomotor domains of hot flashes and night sweats, also showed a significant improvement in VMS for those women treated with the transdermal estradiol spray. While the baseline and week 12 scores were not reported, it was noted that in the 3 spray group, there was a reduction of 1.7 in the hot flush score compared to 0.9 in the placebo group $(P<0.001)$; in the 2 -spray group, there was a reduction of 1.3 compared to 0.9 with placebo $(P=0.018)$; and in the in the 1-spray group, there was a reduction of 1.3 compared to 0.8 with placebo $(P=0.003)$. All of these results were statistically significant.

The most common adverse events reported were headache and breast tenderness. There were no reports of death, pulmonary emboli, venous thromboemboli, myocardial infarction, or stroke. The spray was associated with little to no erythema. Overall, the transdermal estradiol spray was found to be safe and effective in the treatment of VMS. ${ }^{20}$

\section{ET - topical emulsion}

Another alternative to current ET formulations is the micellar nanoparticle estradiol emulsion that is applied as a topical lotion. The emulsion has moisturizing qualities and 
when applied to the skin, forms a drug depot. The estradiol gradually diffuses through the skin and into the blood stream. The pharmacokinetic profile has shown minimal fluctuations in estradiol serum levels. ${ }^{22}$

In a phase III, multicenter, randomized, double-blind, placebo-controlled trial, Simon and the Estrasorb Study group investigated the safety and efficacy of a topical estradiol emulsion. ${ }^{22}$ Postmenopausal women $(\mathrm{n}=200)$ with 120 or more moderate-to-severe hot flashes over a 2-week period were randomly assigned to receive placebo emulsion or estradiol topical emulsion ( $8.6 \mathrm{mg}$ of $17 \beta$-estradiol in $3.45 \mathrm{~g}$ of emulsion) to be applied daily to the thigh and calf of both legs until fully absorbed for 12 weeks. The primary efficacy endpoint was the change in frequency of moderate-to-severe hot flashes from baseline to weeks 4 and 12 . There was a significant decrease in the frequency of hot flashes per day at week $4(P<0.05)$ which continued throughout the treatment period with the peak reduction seen at week $12(-11.1 \pm 6.8$ hot flashes/day for estradiol treatment arm versus $-7.2 \pm 5.4$ for placebo, $P<0.001$ ).

The secondary efficacy endpoint was the change in the severity of hot flashes using a severity score index. Hot flashes were rated as: grade 1 mild, grade 2 moderate, or grade 3 severe. Statistically significant decreases in the daily severity score index was observed at weeks 4,8 and $12(P<0.001)$ for the estradiol treatment arm versus placebo.

The most common adverse events reported in the treatment arm were endometrial/uterine wall thickening, infection, breast pain, application-site reaction, pruritis, vaginal bleeding and vaginal moniliasis. Overall, the estradiol topical emulsion was found to be safe and effective in the treatment of VMS.22

While the estradiol emulsion was found to be safe and effective in the treatment of VMS, in order to have this effect, large doses of the estrogen hormone must be applied topically. This raises concerns as to whether or not the hormone may be transferred to others via skin-to-skin contact. An open-labeled, nonrandomized clinical study performed by Taylor et al investigated the systemic absorption of estradiol in partners of postmenopausal women. Fourteen postmenopausal women and their partners were enrolled in the study. The women applied $1.74 \mathrm{~g}$ of estradiol topical emulsion (containing $2.5 \mathrm{mg}$ of estradiol per gram) to the thigh and calf of both legs for 2 consecutive days. Two hours after application, the male partner rubbed his left forearm vigorously back and forth across the application site for 2 minutes. This was repeated on the male's right forearm 8 hours after application. Serum con- centrations of estradiol, estrone, and estrone sulfate were measured prior to estradiol application and at 1,2 and 8 hours after application for the females, and at 1, 2, 4, 8, 12, 18 and 24 hours after their partners had attempted transfer of the estradiol topical emulsion. Geometric means analysis showed that the area under the serum concentration-time curve from 1 to 8 hours $\left(\mathrm{AUC}_{1-8}\right)$ and the maximum concentration $\left(\mathrm{C}_{\max }\right)$ for estradiol, estrone, and estrone sulfate increased 2- to 3-fold after the second application. These results were statistically significant. Geometric means analysis also showed a significant increase in the area under the serum concentration-time curve from 0-24 hours $\left(\mathrm{AUC}_{0-24}\right)$ and $\mathrm{C}_{\text {max }}$ for estradiol, estrone, and estrone sulfate in the male partners on day 2 when compared to baseline. In particular, the concentration of estradiol increased $25 \%$ from $17.0 \pm 4.3$ to $21.0 \pm 4.4 \mathrm{pg} / \mathrm{mL}$. However, these levels still remained below the upper limit of the normal range for men $(45 \mathrm{pg} / \mathrm{mL}) .{ }^{23}$

\section{Summary}

A variety of estrogen formulations exist for the treatment of menopause-related VMS. Clinical trials have demonstrated that transdermal spray delivery and topical emulsion are both effective methods for this indication and appear to be safe for use in postmenopausal women. In addition, both of these formulations avoid first pass metabolism that may likely result in more consistent absorption, more predictable estrogen concentrations, and easier dosage adjustments when needed. The transdermal estradiol spray was well tolerated by women with headache and breast tenderness being the most commonly reported adverse events. ${ }^{20}$ While the topical estradiol emulsion is effective at decreasing VMS, more studies need to be done to assess skin-to-skin transfer in more detail. The current data is very limited and only observed transfer after two applications. It is unknown what effects the consistent use over time may have on transfer to one's partner. Overall, the transdermal estrogen sprays and topical estradiol emulsion are effective and acceptable alternatives to traditional oral and transdermal patch estrogen used in the treatment of VMS in postmenopausal women.

\section{Disclosures}

The authors declare no conflicts of interest.

\section{References}

1. AACE Menopause Guidelines Revision Task Force. American Association of Clinical Endocrinologists medical guidelines for clinical practice for the diagnosis and treatment of menopause. Endocr Pract. 2006;12:315-337. 
2. North American Menopause Society. Estrogen and progestogen use in peri- and postmenopausal women: July 2008 position statement of The North American Menopause Society. Menopause. 2008;15:584-602.

3. National Institutes of Health. NIH state-of-the-science conference statement on management of menopause-related symptoms. NIH Consens State Sci Statements. 2005;22:1-38.

4. Utian WH. Psychosocial and socioeconomic burden of vasomotor symptoms in menopause: a comprehensive review. Health Qual Life Outcomes. 2005;3:47.

5. North American Menopause Society. Treatment of menopauseassociated vasomotor symptoms: position statement of The North American Menopause Society. Menopause. 2004;11:11-33.

6. Williams RE, Kalilani L, Dibenedetti DB, Zhou X, Fehnel SE, Clark RV. Health care seeking and treatment for menopausal symptoms in the United States. Maturitas. 2007;58:348-358.

7. Freedman RR, Krell W. Reduced thermoregulatory null zone in postmenopausal women with hot flashes. Am J Obstet Gynecol. 1999; 181:66-70.

8. Utian WH. Biosynthesis and physiologic effects of estrogen and pathophysiologic effects of estrogen deficiency: a review. Am J Obstet Gynecol. 1989;161:1828-1831.

9. MacKay HT. Gynecology. In: Tierney LM, McPhee SJ, Papadakis MA, editors. Current Medical Diagnosis and Treatment. San Francisco, CA: Lange Medical Books/McGraw-Hill; 2004:726.

10. Crandall CJ, Crawford SL, Gold EB. Vasomotor symptom prevalence is associated with polymorphisms in sex steroid-metabolizing enzymes and receptors. Am J Med. 2006;119(9 suppl 1):S52-S60.

11. American College of Obstetricians and Gynecologists Women's Health Care Physicians. Vasomotor symptoms. Obstet Gynecol. 2004;104(4 suppl):106S-117S.

12. MacLennan AH, Broadbent JL, Lester S, Moore V. Oral oestrogen and combined oestrogen/progestogen therapy versus placebo for hot flushes. Cochrane Database Syst Rev. 2004;4:CD002978.
13. Nelson HD. Commonly used types of postmenopausal estrogen for treatment of hot flashes: scientific review. JAMA. 2004;291: $1610-1620$

14. Product Information: EVAMIST ${ }^{\mathrm{TM}}$ transdermal spray, estradiol transdermal spray. Vivus, Inc, Mountain View, CA, 2007.

15. Kuhl H. Pharmacology of estrogens and progestogens: influence of different routes of administration. Climacteric. 2005;8 Suppl 1:3-63.

16. Minkin MJ. Considerations in the choice of oral vs transdermal hormone therapy: a review. J Reprod Med. 2004;49:311-320.

17. Rozenbaum H, Birkhäuser M, De Nooyer C, et al. Comparison of two estradiol transdermal systems (Oesclim 50 and Estraderm TTS 50) I. Tolerability, adhesion, and efficacy. Maturitas. 1996;25:161-173.

18. Rozenbaum H, Birkhäuser M, De Nooyer C, et al. Comparison of two estradiol transdermal systems (Oesclim 50 and Estraderm TTS 50). II. Local skin tolerability. Maturitas. 1996;25:175-185.

19. Frenkel Y, Kopernik G, Lazer S, et al. Acceptability and skin reactions to transdermal estrogen replacement therapy in relation to climate. Maturitas. 1994;20:31-36.

20. Buster JE, Koltun WD, Pascual MLG, Day WW, Peterson C. Low-dose estradiol spray to treat vasomotor symptoms. Obstet Gynecol. 2008;111:1343-1351.

21. Schumacher RJ, Gattermeir DJ, Peterson CA, Wisdom C, Day WW. The effects of skin-to-skin contact, application site washing, and sunscreen use on the pharmacokinetics of estradiol from a metered-dose transdermal spray. Menopause. 2009;16:177-183.

22. Simon JA. Estradiol in micellar nanoparticles: the efficacy and safety of a novel transdermal drug-delivery technology in the management of moderate to severe vasomotor symptoms. Menopause. 2006;13:222-231.

23. Taylor MB, Gutierrez MJ. Absorption, bioavailability, and partner transfer of estradiol from a topical emulsion. Pharmaco Therapy. $2008 ; 28: 712-718$
International Journal of General Medicine

\section{Publish your work in this journal}

The International Journal of General Medicine is an international, peer-reviewed open-access journal that focuses on general and internal medicine, pathogenesis, epidemiology, diagnosis, monitoring and treatment protocols. The journal is characterized by the rapid reporting of reviews, original research and clinical studies across all disease areas.

\section{Dovepress}

A key focus is the elucidation of disease processes and management protocols resulting in improved outcomes for the patient.The manuscript management system is completely online and includes a very quick and fair peer-review system. Visit http://www.dovepress.com/ testimonials.php to read real quotes from published authors. 